\title{
Research-based exhibition development: Illustrating the invisible nanoworld
}

\author{
Antti Laherto \\ Department of Education, University of Helsinki, Finland
}

\begin{abstract}
Informal learning environments such as exhibitions in museums and science centres have the potential to promote public engagement in the societally important fields of nanoscience and nanotechnology (NST). This study contributes to research-based development of an NST exhibition by mapping educational, communicational and museographical challenges in illustrating nanoscale science. For the methodological framework, the study employs a previously suggested model based on the Model of Educational Reconstruction. Potential visitors' perspectives were analysed by reviewing research literature on NST learning, and by interviewing science centre visitors. On the basis of the results, the study suggests strategies for illustrating the nanoscale in an exhibition: ways of supporting visitors' scale conceptualisation, presenting images and visualisations deliberately, and using scale models and macroscopic analogies. The study examines how the educational role of science centres may be enhanced by informing exhibition development with visitororiented research.
\end{abstract}

Keywords: exhibition development, model of educational reconstruction, nanoscale, nanoscience education, research-based development, scale conceptualisation, visualisations

Contact: antti.laherto@helsinki.fi

DOI: https:// doi.org/ 10.31129/LUMAT.6.2.323

\section{Introduction}

The fields of nanoscience and nanotechnology (NST) continue to develop rapidly and bring about societally and environmentally significant applications and implications. These emerging fields have also gained growing public interest and media attention. However, and perhaps paradoxically, results of surveys and polls have shown that despite the public's interest in and somewhat positive attitudes towards NST, people's awareness and knowledge of the fields has remained at a rather low level (Crone, 2010; Sahin \& Ekli, 2013; Waldron, Spencer, \& Batt, 2006). Citizens have no firm foundation for understanding NST due to the many conceptual challenges, e.g. concerning relative size of the nanoscale and nanoscale interactions (Schönborn, Höst, \& Lundin Palmerius, 2015). This state of affairs has aroused some concerns, since it is likely that in the near future, citizens will have to make more and more 
decisions on NST-related issues - both at the personal level, as consumers, and also at the societal level (Jones, Blonder, Gardner, Albe, Falvo, \& Chevrier, 2013). Therefore, it has been suggested that some level of understanding of these fields is relevant concerning scientific literacy (Gardner, J ones, Taylor, \& Forrester, 2010; Gilbert \& Lin, 2013; Laherto, 2010; Sabelli et al., 2005; Stevens, Sutherland, \& Krajcik, 2009). In these suggestions, the ambiguous concept of scientific literacy takes a functional and contextualised interpretation, focusing on citizens' ability to identify, to form opinions about and to make reasoned decisions on personal, social, and global issues related to science and technology. Such an emphasis appears in the highly influential PISA definition of scientific literacy (OECD, 2007), recommendations for European science education policies (European Commission, 2015; Osborne \&Dillon, 2008), and “Vision II" for scientific literacy proposed by Roberts (2007; cf. Roberts \& Bybee, 2014). In accordance with these, both the public's awareness of NST and the public's engagement in NST has been called for. In particular, the important ethical issues related to these fields have given rise to the need to engage the public in a deeper discourse on NST and its relations to society (e.g. Cameron \& Mitchell, 2007; J ones et al., 2013).

Consequently, methods and strategies for public communication on NST have been increasingly discussed in the fields of social sciences, science education and science communication (e.g. Gardner et al. 2017; Sweeney \& Seal, 2008). It has been suggested that informal learning environments such as exhibitions in museums and science centres have significant potential not only to educate the public about emerging science and technology, but also to contribute to the science-technologysociety dialogue (Castellini et al., 2007; Crone, 2010; Gilbert \& Lin, 2013; Zenner \& Crone, 2008). Given that such high educational value flow from science centres and museums, the process of developing exhibitions - typically governed by practical and financial aspects and constraints - should beinformed by educational knowledge and expertise. In particular, educational research might support the educational function of those learning environments (see Laherto, 2013, for further discussion).

This paper draws on and expands on a research project that created a design framework ${ }^{1}$ Edelson, 2002) for the development of an exhibition on NST. The purpose

${ }^{1}$ According to Edelson, design frameworks are a type of theory design research can develop. Design frameworks "describe the characteristics that a designed artefact must have to achieve a particular set of goals in a particular context" (Edelson, 2002). 
of the project was to analyse the fields of NST from an educational perspective, in particular from the viewpoint informal learning settings, in order to find wellgrounded approaches for exhibition design. The present paper focuses specifically on the issues related to "the nanoscale". A literature review and an empirical survey were carried out to map the challenges in understanding "the nanoscale", and to find effective approaches for illustrating it in an exhibition. The term "nanoscale" here refers not only to measurement units but essentially also to its objects and phenomena, the tools with which the nanoscale (or the "nanoworld") can be accessed, and the models that describe the phenomena at that scale (cf. Stevens, Sutherland, \& Krajcik, 2009).

Size and scale are only a few of the several educationally significant features of NST. In fact, it can be argued that the most essential ideas - involving the important applications and implications of nanotechnology - involve scale only indirectly (see Kähkönen, Laherto, Lindell, \&Tala, 2016; Laherto, 2011; Laherto, Tirre, Parchmann, Kampschulte \& Schwarzer, in press; further discussion on this follows in the paper). However, since the scale and the smallness of "nano-objects" pose several communicational and museographical challenges regarding exhibition development, they are worth focusing on in this paper.

\section{Framework}

To find research-based guidelines for illustrating the "nanoworld" in an exhibition, the study employed a previously suggested methodological framework (Laherto, 2013) for informing exhibition development through educational research. That framework builds on the Model of Educational Reconstruction (MER) (Duit, 2007). The MER, associated with the design research tradition, combines analytical and empirical educational research with development of practical educational solutions. It consists of three closely interlinked components: 1) analysis of content structure, 2) research on teaching and learning, and 3) design of learning environments. One of the fundamental ideas of the model is that the content structure for instruction cannot be taken directly from science content structure (that is also a human construction), but has to be specially (re)constructed by paying attention to the educational goals, as well as learners' cognitive and affective perspectives (Duit, 2007; Komorek \& Duit, 2004). The methodological framework (Laherto, 2013) adopts the MER for the purpose of informal learning environments. 
The present paper focuses on the second component of the MER, i.e. research on teaching and learning, to find strategies for illustrating NST in an exhibition. However, due to the close interplay between the components in the model, other partstudies are first briefly introduced here. Figure 1 presents content-oriented and visitor-oriented educational research conducted in order to support the choices made in the development of the learning environment.

\begin{tabular}{l}
\hline 1. Content-centred studies \\
- Literature analysis on the \\
educational significance of \\
nanoscale science \\
- Expert surveys on the \\
educational significance of \\
nanoscale science \\
\hline
\end{tabular}

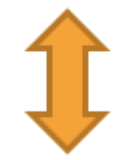

3. Development of learning environment

- Research-based strategies for illustrating nanoscale science in an exhibition

Figure 1. The part-studies of the wider research project, situated within the Model of Educational Reconstruction.

Component 1 of the research project (see Fig. 1), corresponding to the "Analysis of content structure" component in the MER (Duit, 2007), included studies focused on the scientific and technological content of the exhibition, i.e. NST. While there has been a lot of museum research focusing directly on the visitors and their experiences in an exhibition, the absence of content-centred studies in the field of museum education has been pointed out by many (e.g. Gilbert \& Stocklmayer, 2001). In the approach employed in this study, analysis of content structure plays a crucial role in setting the "target" of the exhibition, i.e. the scientific and technological ideas to be presented in the exhibition (Laherto, 2013). The part-studies supporting this process included literature analyses on NST from an educational perspective (Laherto, 2010; Kähkönen et al., 2016), two surveys on science teachers' views on the educational significance of NST (Laherto, 2011; Kähkönen, Laherto, \& Lindell, 2011), and an 
interview study on nanoscientists' views on the nature of NST and its public communication (Laherto et al., in press).

The present paper, instead, focuses on the second component in Fig. 1, corresponding to the "Research on teaching \&learning" component in the MER (Duit, 2007), aimed at understanding the potential visitors' perspectives and learning processes in the context of NST and then using those findings to make recommendations for illustrating the field in an exhibition. In the literature on museum education, knowledge about the audience is nowadays considered equally important to the knowledge of the objects to be presented (Hooper-Greenhill, 1994; Laherto, 2013). A successful audience-responsive approach requires that the staff members do not only rely on their own view when choosing a subject for an exhibition, but that they carefully study the audience's perspectives and interests. As with the content-centred component, the visitor-centred component also involved both theoretical and empirical analyses.

\section{Methods}

The literature analysis on learning the concepts of nanoscale science (Fig. 1) involved science education research literature on teaching and learning the nanoscale concepts, including studies on typical learning difficulties and educational challenges related to this content. A limited (although rapidly growing) amount of such research has been published. The analysis spanned a comprehensive book on NST education (Sweeney \& Seal, 2008) and three review articles (Hingant \&Albe, 2010; J ones et al., 2013; Kähkönen et al., 2016), and all the science education research publications these reviews refers to. A few common themes clearly emerged from the literature, and the analysis focused on these.

The empirical part of the visitor-centred component consisted of a survey in order to get a grasp of potential visitors' perspectives on NST. The survey was conducted in the form of a standardized open-ended interview (Patton, 1990). The sequence of questions is presented in Appendix A. The beginning of the interview aimed to determine thelevel of awareness of the respondent about NST. Since public awareness of these emerging fields was presumed to be quite low, in the latter part of the interview some descriptions were given to the respondents in order to help them to consider the meanings of NST. These descriptions, given to each respondent, are also presented in Appendix A. Furthermore, the aim of the survey was to learn about the 
specific communicational challenges related to the use of visualisations of nanoscale objects. To that end, an image generated with a scanning tunnelling microscope (STM) and a video of a computer simulation were shown to the respondents, with some verbal explanations and questions (see Appendix A). In addition to the interview, the respondents were asked to provide background information in a brief questionnaire: gender, age, educational background, general interest in science, and general interest in technology (the latter questions had a four-point scale "very interested", "quite interested", "not very interested", "not at all interested").

Interviews were carried out in the lobby of the Heureka Finnish science centre. The interviewees were selected randomly from among the adult visitors. The interviews averaged about ten minutes, including completion of the background questionnaire. The number of the interviewees was 28, with 15 women and 13 men. The age of the respondents varied from 20 to 62 years, with a quite even distribution. The educational background varied from secondary school to university level. The great majority (93\%) were at least "quite interested" in both science and technology, as could be anticipated for science centre visitors.

The interviewees' responses were analysed by identifying a few answer categories per question and categorizing the respondents' answers in these categories (Miles \& Huberman, 1994; Patton, 1990). Due to the small sample, no strong generalisations can be made concerning the general public nor even the visitors to the science centre. However, in conjunction with the results of the literature analysis, the results are useful in gaining tentative insight into the awareness and interest of laypersons regarding NST and some idea about the educational and communicational challenges concerning nanoscale issues. In this paper, only the interview results that deals with an understanding of the nanoscale have been reported. Questions about the applications and implications of nanotechnology are beyond the focus of this paper.

\section{$4 \quad$ Results}

\subsection{Literature analysis}

Analysis of the literature on NST teaching and learning revealed that various studies have pointed out quite coherently certain challenges in understanding the nanoscale and its concepts. Several studies have shown that people of all ages have major problems in understanding the scale of NST (Castellini et al., 2007; Tretter, J ones, 
Andre, Negishi, \&Minogue, 2006; Taylor \&J ones, 2008). Furthermore, this does not concern only children and the young; it is natural that all humans - including also scientists working in related fields - have difficulties in conceptualising at the nanoscale because of the change in reasoning it requires (J ones et al., 2013). These challenges are elaborated in what follows, as well as strategies for addressing them.

In their study on the understanding of the size and scale of objects among students (of various ages) and experts, Tretter et al. (2006) concluded (not surprisingly) that students tend to have greater problems with scales for which they have no direct experience, especially microscopic and sub-microscopic scales. Prevalent misunderstandings seem to surround the size of the nanoscale (Schönborn, Höst, \& Lundin Palmerius, 2015). However, the size conceptualisation seems to be easier using relative comparisons than absolute sizes. Taylor and J ones (2008) suggested that by strengthening these relative size perceptions, science education can support qualitative understanding of scale. Quantitative size differences may be added later as mathematical skills develop with age and education. Moreover, size landmarks, or points of reference, seem to be an important tool for anchoring perceptions of the spatial scale (Tretter, 2008). The size of a human appeared to be the clearest reference point, the other common ones being e.g. the width of a hand, the size of an ant, the thickness of a piece of hair or grain of rice, continuing to submicroscopic landmarks like the size of an atom. However, the younger the children are, the lower is their ability to use microscopic and especially the submicroscopic landmarks. In order to solidify these landmarks, education should provide a variety of experiences and reinforcements (Taylor \&J ones, 2008).

An efficient strategy of conceptualising scales that are normally inaccessible to humans, such as the nanoscale, is unitizing. Unitizing means using existing objects to mentally create a new unit that can then be used to measure some other object (for examples, see e.g. Tretter et al., 2006). In order to develop such unitizing skills, science education should provide proportional reasoning abilities (Taylor \& J ones, 2008; Tretter, 2008).

Besides the fact that the scale itself is difficult to comprehend, an additional challenge in NST communication arises because the public does not have a good grasp of the terminology and concepts regarding atoms and molecules and lacks knowledge of the atomic structure of matter (e.g. Crone, 2010). It is common to conceptualize matter as being continuous rather than particulate (Margel, Eylon, \& Scherz, 2008). Children use the terms "atom", "molecule", "cell" ambiguously, and have many 
misconceptions (Murriello, Contier, \& Knobel, 2006; 2009). Additionally, students tend to use "scaling" erroneously and assume that atoms/molecules have the same properties as the macroscopic substance they are part of. The use of macroscopic models for nanoscale phenomena may also contribute to the perception of atoms/molecules as shrunken versions of their macroscopic manifestations (cf. Margel, Eylon, \& Scherz, 2008).

Castellini et al. (2007) argue that one of the fundamental challenges in the public communication of NST is that scientists and also educators tend to assume erroneously that lay people are familiar with the basic ideas of the structure of matter and able to comprehend the size scale. An understanding of nanoscale phenomena, however, can only be built on a comprehension of atoms as building blocks, and the size of them. Therefore, although it may be argued that the most essential ideas of NST involve scale only indirectly, learners need to familiarize themselves with the basics of the scale and the structure of matter before going into actual topics of NST.

Furthermore, the relationships between nanoscale concepts and the observable world can be counterintuitive (e.g. Jones et al., 2013). Since the behaviour of nanoscale particles is governed by quantum effects, discussion of this behaviour in proper terms requires highly sophisticated concepts. This certainly poses educational challenges and the risk of generating misconceptions (Sabelli et al., 2005). Careless simplification of the sophisticated concepts of NST, especially in quantum mechanics, leads to superficiality and the risk of misrepresentation.

The extensive use of images in communicating nanoscale objects and phenomena has recently also become an educational research interest (e.g. Landau, Groscurth, Wright, \& Condit, 2009). The common perception of nanoscience "making atoms visible" is alleged to be problematic (Pitt, 2004), since the microscopy used in nanoscale research is epistemologically not an outright continuation of instruments such as the telescope or light microscope. The scanning force microscope, the atomic force microscope and the scanning tunnelling microscope simply do not portray the visible properties of an object in the sense of geometrical similarity and realistic depiction of colours. Rather, these techniques serve certain theoretical models, but do not generate an empirical database in the same sense as telescopy and light microscopy do (Brune et al., 2006; Pitt, 2004). Brune et al. (2006, pp. 53- 57) also argue that the discourse on NST in general is replete with apparent confusion of models with descriptions of reality due to nanoscientists who tend not to emphasise that their representations are relevant only in the framework of certain theories, 
models, methodological decisions and purposes. Consequently, models are confused with what is being modelled.

In order to learn about the NST-related learning challenges that are specific to exhibitions, publications concerning nano-exhibitions were also searched for to be included in the literature analysis. While several exhibitions on NST topics have been launched in museums and science centres all over the world, there are few publications reporting on the experiences of those projects from an educational viewpoint. When discussing the Brazilian "NanoAventura" exhibition, Murriello, Contier and Knobel (2006; 2009) stress that the most important museographical and communicational challenge in designing exhibits on NST relates to the fact that the objects the fields are based on are invisible to naked eye. Exactly the same notion is stated in the evaluation of "It's a Nanoworld", a travelling exhibition on NST funded by the National Science Foundation in the U.S. (Batt, Waldron, \& Trautmann, 2004). While NanoAventura solved the dilemma of displaying nano-objects in an exhibition by using computer games and virtual representations, "It's a Nanoworld" employed concrete macroscopic models and analogies. In the following, these two approaches among some others are discussed.

On the basis of the literature analysis on the related learning challenges, it can be recommended that an exhibition should provide visitors with opportunities to familiarize themselves with the basics of the scale and the structure of matter before going into actual topics of NST.

\subsection{Visitor survey}

The results of the small survey $(n=28)$ carried out in the lobby of a science centre provided additional insights into the aforementioned findings of the theoretical analysis.

Almost all of the respondents (96\%) had heard of or had at least read something about nanoscience and nanotechnology, with the mass media (newspapers, television and popular science magazines) providing the most important sources of information. The respondents associated NST mostly to physics (71\%), chemistry (43\%) and computer science (25\%), but technology, medicine, astronomy, biology, materials science and mathematics were also mentioned. When asked about their perception of the meaning of "nanoscience and nanotechnology" (question 5, Appendix A), 71\% of the respondents coupled the terms with some kind of "smallness". Every fourth 
interviewee even mentioned the level of atoms or molecules here. On the other hand, $50 \%$ of the respondents associated NST with new technological products, e.g. faster computers, stronger materials and tiny robots.

As the visitor survey was expected to provide additional insight into the educational and communicational challenges discussed in the literature analysis, the questions regarding visitors' perceptions of the scanning tunnelling microscope image $^{2}$ see Appendix A) were of special interest. Firstly, without any explanation, the respondents were asked to interpret what is depicted in the image (question 8). Only $25 \%$ of the interviewees named any nanoscale objects (molecules, atoms etc.), whereas most of the respondents associated the image with either macroscopic objects (35\%) such as "an island" or "a waterdrop" or microscopic objects (29\%) such as "a cell". After the respondents were told that there is a ring of iron atoms on a copper surface and the diameter of the ring is ca. 7 nanometre, $25 \%$ of the respondents knew that the image was created with an electron microscope, whereas $36 \%$ suggested that it was made by computer modelling, without experimental instruments (question 9). After this, the interviewer explained that it was a scanning tunnelling microscope (STM) image, and briefly explained the operating principle of STM, and then asked the respondent to say something about the iron atoms or the copper surface. Even after this attempt for a contextualisation, in question 10 most of the respondents (57\%) came up with false, macroscopic conclusions about the image, for example suggesting that the copper surface is "rough", "soft" or "jelly-like", or that the iron atoms are "sharp" or "rusty", or that "iron is warmer than copper". Still, many respondents reached correct conclusions about the nanostructure, stating e.g. that iron atoms are of equal size and symmetric, or that it is possible to manipulate matter on an atomic scale. In the next question, $43 \%$ suggested that such images could be used in studying the structure of matter or the behaviour of atoms, 28\% said that the STM images are helpful in manipulating matter and developing materials, 7\% mentioned the purposes of communication and popularisation, and $21 \%$ were unable to answer to the question.

In the next phase of the interview, a video of a computer simulation was shown to the interviewees, together with a verbal description as explained in the "Methods" section and in Appendix A (question 12). The respondents were asked to compare the methods and techniques behind the STM image and the simulation. The idea behind

${ }^{2}$ The image shown was of the "quantum corral”, available e.g. at http://www.almaden.ibm.com/vis/stm. 
the question was to see how clear the fundamental difference between these two visualisations, one based on a simulation and the other on empirical methods, is to the respondents. $36 \%$ of the respondents mentioned this difference in some way, while the others responded with some other differences or similarities, e.g. that in the video, the bodies are moving while in the image they are not. $14 \%$ did not provide any answer.

Question 13 proved to be too difficult: $54 \%$ of the respondents could not say anything about it. On the other hand, even $32 \%$ of the respondents were able to provide an answer that is perfectly compatible with the scientific conception, e.g. "the laws change near the atomic level", "at different scales there are different rules". Finally, 68\% of the respondents were interested in learning moreabout NST (question 25). Most of the respondents (71\%) were especially interested in applications, $21 \%$ in scientific results and methods, and 7\% in knowing the risks (question 26).

These results bring out the point that discussing the nanoscale and its phenomena seems like a natural and necessary starting point for the exhibition, although the potential visitors are probably interested in nanotechnological applications too. Special attention is needed when using visualisations of the nanoscale in order to convey the right epistemological ideas with them.

\section{Discussion: strategies for illustrating the nanoscale science in an exhibition}

Based on the results from the above-reported studies, some strategies that could support illustrating nanoscale science in an exhibition are suggested. These presented strategies are all related to "the nanoscale" but they relate to the two aspects of the issue in terms of their goals. Some of the strategies focus on supporting visitors' (geometrical/spatial) scale conceptualisation as such, whereas others address the scale only indirectly. The aim of the latter strategies is to illustrate "the invisible" the nano-sized objects that cannot be observed as such because of their smallness. It is argued that both approaches are needed to help museum visitors to come to grips with nanoscale and its objects. 


\subsection{Illustrating the continuum of scales \& providing size landmarks}

As both the literature review and the empirical survey pointed out, the "smallness" of the nanoscale and its counterintuitive phenomena are very difficult to conceptualise. In education, therefore, they should not be considered in isolation. Instead, an exhibition should guide visitors there by starting from the macroscopic scale, advancing through the microscopic range and finally to the nanoscale. This relative approach may help visitors to construct a continuum of scales and integrate their views of matter across scales. This was also one of the main approaches discussed in the comprehensive workshop reported by Sabelli et al. (2005).

An effective way of displaying this continuum in an exhibition is a scale spectrum with carefully chosen anchoring objects as size landmarks from each scale. Proportional reasoning can be employed by illustrations such as "if a football would be the size of the Earth, then a fullerene would be the size of a football". Besides pictorial presentations, even more effective way of supporting scale conceptualisation is provided by the "powers of ten" videos ${ }^{3}$, recommended also e.g. by Tretter (2008), Castellini (2007) and Sabelli et al. (2005).

If a visitor understands the linear scale continuum from the macroscopic world to the nanoscale, it does not yet mean that (s)he has an understanding of any of the key ideas of NST, such as the size-dependent properties of matter. However, the visitor has a good foundation on which to situate later insights of nanoscale objects and phenomena.

\subsection{Using images and visualisations}

The research reviewed for this paper showed that personal experiences are essential in understanding scales. As discussed above, people have major difficulties in conceptualising size scales which they do not have experience of. Since it is not possible to obtain direct experiences at the nanoscale, and quantum phenomena cannot be replicated at the macroscale, images, visualisations and simulations must be used instead. Furthermore, several studies pointed out that visual models are crucial in students' understanding of sophisticated concepts (see e.g. Tretter, 2008). Therefore, abstract nanoscale concepts should also be taught with linkage to pictorial representations.

\footnotetext{
${ }^{3}$ There are many popular videos available, see e.g. http://www.powersof10.com.
} 
Accordingly, images and visualisations are used extensively in NST communication. These methods are also natural for museums and science centres e.g. virtual representations have been common in science museums for a long time (see Hooper-Greenhill, 1994). For an example of an NST exhibition relying completely on virtual representations, see Murriello, Contier, \& Knobel (2009).

The power of visual representations in communicating NST also entails pitfalls. The public's understanding of these images and the impact of the images on the public's perceptions has become a research interest (e.g. Landau et al., 2009). The literature review found several articles that presented discussion of the risks of causing misconceptions - for example, it is questionable indeed what "seeing atoms" is by using a scanning tunnelling microscope (STM) or an atomic force microscope (AFM) (cf. Pitt, 2004; Robinson, 2004). Our empirical findings support the conclusion of the literature review, implying that special attention should be paid when communicating the nanoscale using such images, in order to avoid misleading learners into false models of direct sense perception and epistemological misunderstandings.

\subsection{Using scale models and analogies to macroscopic objects}

Another strategy for illustrating nanoscale objects in an exhibition is to use macroscopic scale models and analogies. They are popular in public communication of NST, especially in models of the structure of matter (with macro objects modelling atoms and molecules for example), as well as in macroscopic models of electron microscopy (e.g. "LEGO-AFM", see Sabelli et al., 2005). An American exhibition on NST entitled "It's a Nano World" relied solely on macroscopic analogies and "enlargement models" (Batt et al., 2004).

These models and analogies are powerful tools for anchoring the issues in learners' everyday experiences. This is especially crucial in informal learning environments: because of the free-choice-learning nature of them, it is a necessity to address visitors' needs and interests in an exhibition in order to gain any contact. Therefore, macroscopic points of comparison should be chosen so that they are relevant to visitors.

Demonstrating nanoscale phenomena by using macroscopic analogies is tempting indeed. It should be noted, however, that they do not reflect the discontinuous change of properties at a certain size, or any other quantum phenomena. Consequently, there 
is a major risk of causing misconceptions and, even, contradicting the major learning goal: properties of objects change discontinuously at a certain size. This important learning goal may become blurred when objects of a macroscopic nature and behaviour are used to demonstrate the nanoscale phenomena that do not obey macro laws. Due to these concerns, exhibits of this kind should be evaluated before being used in an exhibition, in order to find out the potential misconceptions they may generate. The nature and the limitations of the analogy should be pointed out.

Still, analogical models may be especially helpful in illuminating "scaling effects" (as suggested by Taylor and J ones, 2008). These effects mostly follow from the simple and classically understood way how a change in the size of an object affects the ratio of its surface area to volume. In studies on reasoning patterns, it has been found out that students find understanding scaling effects to be challenging. Macroscopic analogies may help this: for an example, surface-area-to-volume experiments with differently sized pieces of ice to illustrate heat loss.

\subsection{Accessing nanoscale by instruments}

Instead of drawing solely on visualisations and macroscopic analogies, it is both useful and possible to provide visitors with a "real" access to nanoscale phenomena, for example by using a scanning tunnelling microscope (STM) or an atomic force microscope (AFM) (also suggested by Sabelli et al., 2005). By using real instruments to make measurements on real nanoscale samples may support visitors' understanding of the connection of the nanometric world to its manifestations and representations in the macroscopic world. Reasonably-priced instruments are available for educational purposes, and applications for remote access to an AFM placed in a university laboratory are also available. Furthermore, the use of a haptic interface has shown promise in visitors' learning about molecular interactions (Bivall, Ainsworth, \& Tibell, 2011). These methods have even been used in classrooms (see e.g. Fraundorf \& Liu, 2008; Jones, 2008), and the resources are better again in museums. 


\section{Conclusion}

Informal learning environments have a significant potential to contribute to public understanding and engagement in emerging fields of science and technology such as NST. The study presented here connects to a wider project on research-based development of such settings (Laherto, 2013). The Model of Educational Reconstruction (Duit, 2007; Komorek \& Duit, 2004) has been used as the basis, drawing on both content analysis of the subject matter and studies on learners' perspectives. In this paper, educational, communicational and museographical issues related to the scale of NST have been scrutinised in order to find well-grounded strategies for exhibition development. The challenges and the recommended strategies are summarised in Table 1.

Table 8. Challenges in illustrating the nanoworld, and corresponding strategies for exhibition development.

\begin{tabular}{|c|c|c|}
\hline Challenge & References & Recommended strategies \\
\hline $\begin{array}{l}\text { invisible nano-objects: challenge to } \\
\text { the "presence culture" in exhibitions }\end{array}$ & $\begin{array}{l}\text { Batt, Waldron, \& Trautmann, } \\
\text { 2004; Murriello, Contier, \& } \\
\text { Knobel, 2006; 2009; Taylor \& } \\
\text { Jones, } 2008\end{array}$ & $\begin{array}{l}\text { - macroscopic models and } \\
\text { analogies (especially in } \\
\text { illustrating scaling } \\
\text { effects) } \\
\text { - computer games and } \\
\text { virtual representations } \\
\text { - shifting to the "meaning } \\
\text { culture": societal } \\
\text { significance of NST }\end{array}$ \\
\hline $\begin{array}{l}\text { no experience of sub-microscopic } \\
\text { scales }\end{array}$ & $\begin{array}{l}\text { Bivall, Ainsworth, \& Tibell, } \\
\text { 2011; Fraundorf \& Liu, 2008; } \\
\text { Jones, 2008; Murriello, Contier, } \\
\text { \& Knobel, 2009; Sabelli et al., } \\
\text { 2005; Tretter et al., 2006; } \\
\text { Tretter, } 2008\end{array}$ & $\begin{array}{l}\text { - images, visualisations } \\
\text { and simulations } \\
\text { - real access to nanoscale } \\
\text { with instruments } \\
\text { (remote or actual } \\
\text { educational AFM/STM) } \\
\text { - haptic interfaces }\end{array}$ \\
\hline difficulties in scale conceptualisation & $\begin{array}{l}\text { Castellini et al., 2007; Sabelli et } \\
\text { al., 2005; Schönborn, Höst, \& } \\
\text { Lundin Palmerius, 2015; } \\
\text { Taylor \& Jones, 2008; Tretter, } \\
\text { 2008; Tretter et al., } 2006\end{array}$ & $\begin{array}{l}\text { - } \text { relative comparisons } \\
\text { - } \text { instead of absolute sizes landmarks } \\
\text { - continuum of scales }\end{array}$ \\
\hline difficulties in proportional reasoning & $\begin{array}{l}\text { Castellini et al., 2007; Sabelli et } \\
\text { al., 2005; Tretter et al., 2006; } \\
\text { Taylor \& Jones, 2008; Tretter, } \\
2008\end{array}$ & $\begin{array}{l}\text { - unitizing } \\
\text { - proportional illustrations } \\
\text { - "powers of } 10 \text { " videos }\end{array}$ \\
\hline
\end{tabular}


difficulties in understanding sizedependent properties

misconceptions: matter as continuous rather than particulate

misconception: atoms/molecules having the same properties as the macroscopic substance

risks of images and visualisations: epistemological misunderstandings, false models of direct sense perception
Sabelli et al., 2005; Taylor \& Jones, 2008

Castellini et al., 2007; Crone, 2010; Margel, Eylon, \& Scherz, 2008; Murriello, Contier, \& Knobel, 2006; 2009

Jones et al., 2013; Margel, Eylon, \& Scherz, 2008; Sabelli et al., 2005

Landau et al., 2009; Brune et al., 2006; Pitt, 2004; Robinson, 2004

Batt et al., 2004; Brune et al., 2006; Sabelli, 2005
- illustrations on surfacevolume ratio

- multiple examples and representations

- discussing the structure of matter

- illustrating size and scale

- discussing counterintuitive quantum effects

- explaining the methodological and epistemological issues

- careful front-end evaluation of visualisations for potential misconceptions

- discussing scientific modelling

- pointing out the limitations of analogies

- careful front-end evaluation of exhibit models for potential misconceptions

In general, exhibitions seem to fit well as learning environments on NST. Contrary to formal education, in museums and science centres, there are no disciplinary boundaries or other curriculum constraints that do not cohere with the interdisciplinary nature of NST (cf. Kähkönen et al., 2016). Also, the instrumentation required for experimental work and "seeing" invisible nano-objects may be unattainable for classroom purposes, but the resources are better in museums and science centres. Moreover, given the needs discussed in the Introduction, exhibitions can provide a quick response to the growing public interest. Yet, informal learning environments such as exhibitions certainly bear some additional educational challenges too. Because of the fragmental nature of the learning environment, it is difficult to learn structured information at an exhibition. This poses major challenges for conceptual learning, considering also complex and sophisticated concepts and 
knowledge structure of NST. Moreover, due to the diversity of learners in a science centre, it is complicated to take visitors' preconceptions and other perspectives into account in exhibition development.

The axiomatic fact that nano-objects are neither visible nor tangible constitutes an interesting museographical challenge. In the traditional view, it makes nanoscale science a problematic topic for a museum, since it does not fit in with the idea of materiality and "presence culture" that is considered the essence of museums (Söderqvist, Bencard, \&Mordhorst, 2009). Nanoscale objects cannot be collected and displayed in an exhibition as such. An exhibition can include NST instruments and macroscopic objects that nanoparticles are part of, showing the macroscopic manifestations of nanoscale phenomena. Such exhibits, however, do not present the scientific essence (for a discussion on the same issue in the context of biomedicine, see Söderqvist, Bencard, \& Mordhorst, 2009). Yet, this museographical challenge of the "presence culture" is even wider and concerns all contemporary fields of science in which social and cultural aspects are typically deeply connected to the "scientific content". Representing these social and cultural phenomena in an exhibition cannot be done in traditional museographical ways, since they usually do not manifest themselves through material artefact. This development has shifted the focus of museums from "presence culture" towards "meaning culture" (Söderqvist, Bencard, \& Mordhorst, 2009). Due to the important social implications of nanoscience and nanotechnology, and the limited opportunities for "presence effects", focusing on the meanings seems like a reasonable starting point for a nanoscience exhibition. Accordingly, the study reported here focused on the communication of one of the meanings of NST, i.e. an understanding of the nanoscale. Given the societal and educational significance of NST, it should be acknowledged that social and cultural meanings are at least equally important aspects of an exhibition.

In this paper, it has been argued that scale-related issues are a natural starting point for development of an informal learning environment on NST. Despite several educational challenges highlighted by the study, there are reasonable strategies to illustrate the nanoscale and its objects in an exhibition. Supporting visitors' scale conceptualisation by presenting scales as a continuum with size landmarks, using images and visualisations, as well as using macroscopic models and analogies (only in the context of scaling effects!) are effective tools for that. However, each of these approaches also entail some pitfalls, so they should be used only deliberately. Also, it should be noted that an exhibition should not focus too much on the scale itself, but 
on the properties of matter that are the essence of NST. If visitors only learn to "scale down" their macroscopic experiences, they will end up with wrong conclusions about the nanoscale. The discontinuously changing properties of matter should be kept in mind.

Indeed, it remains debatable if size and scale itself is in fact the very essence of NST. Either way, when taking an educational perspective, size and scale are definitely important. Scale conceptualisation is an important interdisciplinary theme of science education in general, and also plays a significant role in scientific literacy (as defined in the introduction; see also Gardner et al., 2010; Kähkönen et al., 2016; Tretter, 2008). It has also been suggested as one of the "Big Ideas of Nanoscience", and its incorporation in school curricula has been recommended (Sabelli et al., 2005; Stevens et al., 2009). Thereby the conceptualisation of scale is nevertheless a natural starting point for public communication and informal education in NST.

The guidelines suggested in the paper can be considered as a design framework (Edelson, 2002) for the development of such learning environments. Creating a prescriptive, generalized set of design guidelines such as the one in this study is a typical theoretical outcome of design-oriented research. Besides being applied in the research project this study is connected with, the results could also be used in research and development on other educational solutions.

\section{References}

Batt, C., Waldron, A., \&Trautmann, C. (2004). It's a nanoworld: A study of use. Retrieved from http:/ / eduinc.org// ItsaNanoWorldSummativeStudy.pdf

Bivall, P., Ainsworth, S., \& Tibell, L. (2011). Do haptic representations help complex molecular learning? Science Education, 95(4), 700- 719.

Brune, H., Ernst, H., Grunwald, A., Grünwald, W., Hofmann, H., Krug, H., et al. (2006). Nanotechnology. assessment and perspectives. Berlin Heidelberg: Springer.

Cameron, N., \&Mitchell, M. (2007). Nanoscale: Issues and perpectives for the nano century. Hoboken, NJ : J ohn Wiley \& Sons.

Castellini, O., Welejko, G., Holladay, C., Theim, T., Zenner, G., \& Crone, W. (2007). Nanotechnology and the public: Effectively communicating nanoscale science and engineering concepts. J ournal of Nanoparticle Research, 9(2), 183-189.

Crone, W. (2010). Bringing nano to the public: A collaboration opportunity for researchers and museums. J ournal of Nano Education, 2(1-2), 102-116.

Duit, R. (2007). Science education research internationally: Conceptions, research methods, domains of research. Eurasia J ournal of Mathematics, Science \& Technology Education, 3(1), 3-15.

Edelson, D. (2002). Design research: What we learn when we engage in design. J ournal of the Learning Sciences, 11(1), 105. 
European Commission (2015). Science Education for Responsible Citizenship. Report to the European Commission of the expert group on science education. Luxembourg: Publications Office of the European Union.

Fraundorf, P., \&Liu, J . (2008). Microscopic perspectives on informal, introductory, and industry nanochallenges. In A. Sweeney, \& S. Seal (Eds.), Nanoscale science and engineering education (pp. 167-183). Stevenson Ranch, CA: American Scientific Publishers.

Gardner, G., J ones, G., Albe, V., Blonder, R., Laherto, A., Macher, D., \& Paechter, M. (2017). Factors Influencing Postsecondary STEM Students' Views of the Public Communication of an Emergent Technology: a Cross-National Study from Five Universities. Research in Science Education, 47(5), pp. 1011-1029. https:/ / doi.org/ 10.1007/ s11165-016-9537-7

Gardner, G., J ones, G., Taylor, A., \& Forrester, J . (2010). Students' risk perceptions of nanotechnology applications: Implications for science education. International J ournal of Science Education, 32(14), 1951-1969.

Gilbert, J ., \& Lin, H. (2013). How might adults learn about new science and technology? The case of nanoscience and nanotechnology. International J ournal of Science Education, Part B: Communication and Public Engagement, 3(3), 267-292.

Gilbert, J ., \& Stocklmayer, S. (2001). The design of interactive exhibits to promote the making of meaning. Museum Management and Curatorship, 19(1), 41-50.

Hingant, B., \& Albe, V. (2010). Nanosciences and nanotechnologies learning and teaching in secondary education: a review of literature. Studies in Science Education, 46(2), 121-152.

Hooper-Greenhill, E. (1994). The educational role of the museum. London: Routledge.

J ones, G. (2008). Exploring nanoscale science with middle and high school students. In A. Sweeney, \& S. Seal (Eds.), Nanoscale science and engineering education (pp. 81-89). Stevenson Ranch, CA: American Scientific Publishers.

J ones, G., Blonder, R., Gardner, G., Albe, V., Falvo, M., \& Chevrier, J . (2013). Nanotechnology and Nanoscale Science: Educational challenges. International J ournal of Science Education, 35(9), 1490-1512.

Komorek, M., \& Duit, R. (2004). The teaching experiment as a powerful method to develop and evaluate teaching and learning sequences in the domain of non-linear systems. International J ournal of Science Education, 26(5), 619-633.

Kähkönen, A., Laherto, A., \& Lindell, A. (2011). Intrinsic and extrinsic barriers to teaching nanoscale science: Finnish teachers' perspectives. J ournal of Nano Education, 3(1), pp. 112.

Kähkönen, A., Laherto, A., Lindell, A., \& Tala, S. (2016). Interdisciplinary Nature of Nanoscience: Implications for Education. In K. Winkelman, \&B. Bhutan (Eds.), Global Perspectives of Nanoscience and Engineering Education (pp. 35-81). Science Policy Reports. Switzerland: Springer.

Laherto, A. (2010). An analysis of the educational significance of nanoscience and nanotechnology in scientific and technological literacy. Science Education International, 21(3), 160-175.

Laherto, A. (2011). Incorporating nanoscale science and technology into secondary school curriculum: Views of nano-trained science teachers. Nordic Studies in Science Education, $7(2), 126-139$.

Laherto, A. (2013). Informing the development of science exhibitions through educational research. International J ournal of Science Education, Part B: Communication and Public Engagement, 3(2), 121-143.

Laherto, A., Tirre, F., Parchmann, I., Kampschulte, L., \& Schwarzer, S. (in press). Scientists' perceptions on the nature of nanoscience and its public communication. Accepted to be published in Problems of Education in the 21st Century. 
Landau, J ., Groscurth, C., Wright, L., \& Condit, C. (2009). Visualizing nanotechnology: The impact of visual images on lay American audience associations with nanotechnology. Public Understanding of Science, 18(3), 325-337.

Margel, H., Eylon, B., \& Scherz, Z. (2008). A longitudinal study of junior high school students' conceptions of the structure of materials. J ournal of Research in Science Teaching, 45(1), 132-152.

Miles, M., \&Huberman, A. (1994). Qualitative data analysis (2nd ed.). Thousand Oaks, CA: SAGE Publications.

Murriello, S., Contier, D., \&Knobel, M. (2006). Challenges of an exhibit on nanoscience and nanotechnology. J ournal of Science Communication, 5(4), 1.

Murriello, S., Contier, D., \& Knobel, M. (2009). NanoAventura: An interactive exhibition on nanoscience and nanotechnology as an educational tool. J ournal of Nano Education, 1, 96105.

OECD (2007). Assessing scientific, reading and mathematical literacy: A framework for PISA 2006. Paris: OECD Publications.

Osborne, J ., \& Dillon, J . (2008). Science Education in Europe: Critical Reflections. A Report to the Nuffield Foundation. London: The Nuffield Foundation.

Patton, M. (1990). Qualitative evaluation and research methods. Newbury Park, CA: Sage Publications.

Pitt, J . (2004). The epistemology of the very small. In D. Baird, A. Nordmann \&J . Schummer (Eds.), Discovering the nanoscale (157-164). Amsterdam: IOS Press.

Roberts, D. A. (2007). Scientific literacy/ Science literacy. In S. K. Abell, \& N. G. Lederman (Eds.), Handbook of research on science education (pp. 729-780). Mahwah, NJ : Lawrence Erlbaum Associates.

Roberts, D. A., \& Bybee, R. W. (2014). Scientific literacy, science literacy, and science education. In N. G. Lederman, \& S. K. Abell (Eds.), Handbook of research on science education (vol. 2) (pp. 545-558). New York, NY: Routledge.

Robinson, C. (2004). Images in NanoScience/Technology. In D. Baird, A. Nordmann \&J . Schummer (Eds.), Discovering the nanoscale (pp. 165-169). Amsterdam: IOS Press.

Sabelli, N., Schank, P., Rosenquist, A., Stanford, T., Patton, C., Cormia, R., \&Hurst, K. (2005). Report of the workshop on science and technology education at the nanoscale. Draft Technical Report. Menlo Park, CA: SRI International.

Sahin, N., \&Ekli, E. (2013). Nanotechnology awareness, opinions and risk perceptions among middle school students. International J ournal of Technology and Design Education, 23(4), 867-881.

Schönborn, K., Höst, G. \&Lundin Palmerius, K. (2015). Measuring understanding of nanoscience and nanotechnology: development and validation of the nano-knowledge instrument (NanoKI). Chemistry Education Research and Practice, 16(2), 346-354.

Stevens, S., Sutherland, L., \& Krajcik, J . (2009). The big ideas of nanoscale science and engineering. Arlington, VA: NSTA Press.

Sweeney, A. E., \& Seal, S. (Eds.) (2008). Nanoscale science and engineering education. Stevenson Ranch, CA: American Scientific Publishers.

Söderqvist, T., Bencard, A., \& Mordhorst, C. (2009). Between meaning culture and presence effects: Contemporary biomedical objects as a challenge to museums. Studies in History and Philosophy of Science, 40(4), 431-438.

Taylor, A. \&J ones, M. G. (2008). Proportional reasoning ability and concepts of scale: Surface area to volume relationships in science. International J ournal of Science Education, 31(9), 1231- 1247. 
Tretter, T. (2008). Nanoscience and conceptions of size and scale in precollege education. In A. E. Sweeney, \& S. Seal (Eds.), Nanoscale science and engineering education (pp. 149-166). CA: American Scientific Publishers.

Tretter, T., J ones, M., Andre, T., Negishi, A., \& Minogue, J . (2006). Conceptual boundaries and distances: Students' and experts' concepts of the scale of scientific phenomena. J ournal of Research in Science Teaching, 43(3), 282.

Waldron, A., Spencer, D., \& Batt, C. (2006). The current state of public understanding of nanotechnology. J ournal of Nanoparticle Research, 8(5), 569-575.

Zenner, G., \& Crone, W. (2008). Introducing nanotechnology and society issues into the classroom. In A. E. Sweeney, \& S. Seal (Eds.), Nanoscale science and engineering education (pp. 622-647). Stevenson Ranch, CA: American Scientific Publishers. 


\section{Appendix A: Questions of the interview: science centre visitors' perspectives on nanoscience and nanotechnology (translated from Finnish by the author)}
4. Have you heard or read about nanoscience or nanotechnology (NST)?
5. Where did you hear/ read about NST?
6. Have you studied NST, or has your work experience concerned these fields?
7. In your opinion, what fields of science is NST related to?
8. In your opinion, what does NST mean?

When the respondent has responded to question 5, the interviewer provides a simple definition of NST: "Nanoscience and nanotechnology concern the research, manipulation and construction of very small structures. According to a common definition, the structures of NST are in the size range of 1-100 nanometres, at least in one dimension (length, breadth or thickness). A nanometre is one millionth of a millimetre. This means that the structures of NST can be as small as a few molecules or atoms. At this scale, matter gains new properties that depend on size. These properties can, for instance, be mechanical, electrical or optical.”

9. Do you know any applications or products that exploit nanotechnology?

10.Here I have talked about nanoscience and nanotechnology. Do you think that there is a difference between them?

The interviewer shows a scanning tunnelling microscope (STM) image of a nanoscale structure. ["Quantum corral”, image originally created by IBM Corporation and available at the STM Gallery, http://www.almaden.ibm.com/vis/stm/gallery.html. The image is also on the cover of the printed version of this dissertation.]

\section{What do you think is presented in this image?}

After the respondent has answered question 8, the interviewer explains that there is a copper surface, in which a ring is constructed out of single iron atoms. The diameter of the ring is ca. $7 \mathrm{~nm}$.

\section{How and with which instruments was this image created?}

The interviewer explains that the image was created with a scanning tunnelling microscope. STM has a sharp tip that is slowly moved across the surface, measuring the properties of the surface.

13. On the basis of this image, what can you say about the iron atoms or the copper surface?

14. For what purpose do you think images of this kind can be used in nanoscience? 
The interviewer shows a computer simulation and gives the following explanation: "In this computer simulation a small, spherical carbon structure collides with a tubular carbon structure.”

15. When you think of the creation of this video and the recent image, what similarities and differences come to your mind?

After the respondent has answered question 12, the interviewer explains: "The carbon nanotube shown in the video is one important structure studied and applied in NST. It has interesting properties: a carbon nanotube is extremely strong, and it conducts electricity and heat very well. It is a good example of a central idea of NST: below a certain size, matter may exhibit totally new and even revolutionary properties.”

16. What do you think these new properties result from?

17. Generally speaking, what potential benefits do you think will follow from nanotechnology?

18. What disadvantages and risks will follow from nanotechnology?

19. Which do you consider more significant, the benefits or the disadvantages/ risks?

After the respondent has answered question 16, the interviewer says: "Finally, I will read some statements. Please respond on the scale $1 . . .5$ depending on how much you agree with the statement. ' 1 ' means you do not agree at all, and ‘5’ means you completely agree. You can also respond 'I cannot say'.”

20. The general public should be heard when making decisions about the development of NST.

21. Decisions on NST should be made on the basis of expert views and advice.

22. Decisions on NST should be made on the basis of views of average citizens.

23. Decisions on NST should be based on scientific knowledge of the risks and benefits.

24. Decisions on NST should be based on moral and ethical considerations.

25. Citizens should be told about NST and be able to decide independently whether they want to use products developed with these methods.

26. Although nanotechnology may bear some unknown risks, it is an inevitable part of our future, so we should just make sure that it is used as safely as possible.

27. NST should be regulated and supervised more strictly than before.

28. I am interested in knowing more about NST.

29. What interests you the most about NST? 